\title{
GENERALIZING THE ALEXANDROFF-URYSOHN DOUBLE CIRCUMFERENCE CONSTRUCTION
}

\author{
RICHARD E. CHANDLER, GARY D. FAULKNER,
} JOSEPHINE P. GUGLIELMI AND MARGARET C. MEMORY

\begin{abstract}
This paper is a unification of the "Alexandroff-Urysohn Double Circumference Construction", the one-point compactification, the Steiner and Steiner remainder theorem, and Whyburn's Unified Space. All of these are shown to be different aspects of a single construction.
\end{abstract}

The celebrated double circumference of Alexandroff and Urysohn was originally described in [1, pp. 13-15]. It is constructed by taking the union of two concentric circles and choosing the topology on the outer circle to be discrete. On the inner circle a basic neighborhood of a point $x$ is defined to be the union of an open arc on the inner circle containing $x$ and the corresponding (by radial projection) open arc on the outer circle with the point which corresponds to $x$ deleted.

Engelking [4] generalized this construction by defining a similar topology on the union of two sets in the case where the first has a compact topology and the second is the same set but with the discrete topology.

In the examples above, the spaces constructed are compact Hausdorff spaces each of which contains a discrete set as a dense subset. From our point of view the examples provide us with a means of obtaining compactifications of these discrete spaces. Recall that a compactification of a locally compact space $X$ is a compact Hausdorff space $Z$ such that $X$ is embedded densely in $Z . Z \backslash X$ is then called a remainder of $X$.

In this paper we develop a method of compactification which generalizes the above examples. We also show how this method of construction relates to one developed by Steiner and Steiner [5] and to Whyburn's Unified Space [6].

We would like this method to give a compactification of an arbitrary (not necessarily discrete) locally compact space. We first note that both the previous constructions require a correspondence between points in the space $X$ which was compactified and points in the remainder $Y$. In our generalization this correspondence will be given by a continuous map $f: X \rightarrow Y$.

Mimicking Engelking we describe the topology on $X \cup Y$ as follows: For a point $p$ in $X$, basic neighborhoods in $X \cup Y$ will be the neighborhoods of $p$ in the original topology on $X$. For a point $q$ in $Y$ and $U$ an original neighborhood of $q$ in $Y$ we start with $f^{-1}(U) \cup U$ and make the following observations.

Received by the editors December 19, 1980.

1980 Mathematics Subject Classification. Primary 54D35, 54D40.

Key words and phrases. Compactification, remainder, singular map, unified space. $0002-9939 / 81 / 0000-0538 / \$ 01.75$ 
(1) So that $X \cup Y$ will be Hausdorff, whenever $f(p)=q$ we must delete some neighborhood of $p$.

(2) So that $X \cup Y$ will be compact, what we delete must be compact.

(3) So that $X$ is dense in $X \cup Y$, we must be assured that $f^{-1}(U)$ is not contained in any compact neighborhoods that are deleted. In particular, we must require that $\overline{f(X)}=Y$.

Specifically, for $q \in Y$ we define a basic neighborhood of $q$ to be $\left(f^{-1}(U) \backslash F\right)$ $U U$ where $U$ is any neighborhood of $q$ in the original topology on $Y$, and $F$ is any compact set in $X$. However from (3) above we see that if we want $X$ to be dense in $X \cup Y$ then $\overline{f^{-1}(U)}$ must not be contained in any compact set.

This leads us to a concept which has been much studied in the past in a different context.

The singular set $\mathcal{S}(f)$ may be defined by $\mathcal{S}(f)=\left\{y \in Y \mid \overline{f^{-1}(U)}\right.$ is not compact for any open $U \subseteq Y$ with $y \in U\}$. For our construction above to yield a compactification of $X$, we need $\delta(f)$ to be $Y$. Note that this also implies $\overline{f(X)}=Y$. See Whyburn [7], Cain [2], or Cain, Chandler, Faulkner [3] for additional material on $\delta(f)$.

We note the following properties of this construction:

(1) $\mathrm{Cl}_{X \cup Y}(X)=X \cup \delta(f)$. For suppose $x \in \mathrm{Cl}_{X \cup Y}(X)$. If $x \notin X$,

$$
\left[\left(f^{-1}(U) \backslash F\right) \cup U\right] \cap X=f^{-1}(U) \backslash F \neq \varnothing
$$

for all neighborhoods $U$ of $x$ in $Y$ and all compact $F$ in $X$. Then $\overline{f^{-1}(\bar{U})}$ is not compact, and $x \in S(f)$.

Conversely, let $x \in X \cup \mathcal{S}(f)$. For $x \in \mathcal{S}(f), f^{-1}(\bar{U})$ is not compact for all neighborhoods $U$ of $X$ in $Y$, so $f^{-1}(\bar{U}) \backslash F \neq \varnothing$ for all compact $F$ in $X$. Every neighborhood of $x$ intersects $X$, so $x \in \mathrm{Cl}_{X \cup Y}(X)$.

(2) Given a space $X$, a compact space $Y$, and a mapping $f: X \rightarrow Y$ with $\delta(f)=Y, X \cup Y$ is a compactification of $X$.

It is clear that the radial projection map of the Alexandroff-Urysohn construction and the formal identity in Engelking's construction are singular maps (so long as the compact space has no isolated points) and that the topology in their constructions is the same as that induced by our definition.

Furthermore, if we define $f(x)=\omega$ for each $x \in X$, then our topology on $X \cup\{\omega\}$ is the same as the 1-point compactification of $X$, since the neighborhoods of $\omega$ in this case are $(X \backslash F) \cup\{\omega\}$ where $F$ is an arbitrary compact set.

Let $X^{*}=X \cup\{\omega\}$ denote the 1-point compactification of $X$ and let $N(\omega)$ denote a neighborhood of $\omega$. Steiner and Steiner [5] showed that if $X$ is locally compact, $K$ is compact, and if there is a continuous map $f: X \rightarrow K$ such that $f[N(\omega) \cap X]$ is dense for each neighborhood $N(\omega)$, of $\omega$, then $X$ has a compactification $\hat{X}$ with a remainder homeomorphic to $K$. The closure of the graph of $f$ in $X^{*} \times K$ was shown to be such an $\hat{X}$.

The density requirement on $f[N(\omega) \cap X]$ is equivalent to our requirement that $\delta(f)=K$. So from the conditions of the Steiner-Steiner theorem, our method produces a compactification $\tilde{X}$ of $X$. The two compactifications are in fact equivalent. That is, if $h$ and $m$ are the embeddings of $X$ into $\hat{X}$ and $\tilde{X}$, there is a 
homeomorphism $l$ from $\hat{X}$ to $\tilde{X}$ such that $l \circ m(x)=h(x)$ for all $x \in X$. Such an $l$ may be defined by

$$
\left\{\begin{array}{c}
l(x)=(x, f(x)) \text { for } x \in X, \\
l(y)=(\omega, y) \text { for } y \in K .
\end{array}\right.
$$

The example $f(x)=\sin (x)$ on $X=[0, \infty)$ provides an easily visualized space for observing the above constructions, giving $[-1,1]$ as a remainder.

Our method also indicates a connection between the Steiner-Steiner method and Whyburn's Unified Space which is otherwise not evident.

Given a map $f: X \rightarrow Y$ where $X$ and $Y$ are $T_{1}$ spaces, Whyburn [6], [7] defined the unified space to be $X \cup Y$ with the following topology: $0 \subset X \cup Y$ is open if

(i) $0 \cap Y$ is open in $Y, 0 \cap X$ is open in $X$;

(ii) for any compact $K \subseteq 0 \cap Y, f^{-1}(K) \cap(X \backslash 0)$ is compact.

If $f$ is singular $(\delta(f)=Y)$ and $Y$ is locally compact, Whyburn's topology on $X \cup Y$ is the same as ours.

Let $\left(f^{-1}(U) \backslash F\right) \cup U=0$ be a basic set in our topology. Then $0 \cap Y=U$ is open in $Y$ and $0 \cap X=f^{-1}(U) \backslash F$ is open in $X$. Let $K$ be a compact set contained in $U$. Since $f^{-1}(K) \subseteq f^{-1}(U)$,

$$
f^{-1}(K) \cap(X \backslash 0)=f^{-1}(K) \cap\left[X \backslash\left(f^{-1}(U) \backslash F\right)\right]=f^{-1}(K) \cap F .
$$

$f^{-1}(K) \cap F$ is a closed set contained in the compact set $F$ so it is compact. Therefore, 0 is open in the Whyburn topology.

Let 0 be an open set in the Whyburn topology. Let $p \in 0.0 \cap X=V$ is open in our topology so if $p \in 0 \cap X$, then $p \in V \subseteq 0$. If $Y$ is locally compact and $p \in 0 \cap Y$, there is a $U$ open in $Y, p \in U \subseteq \bar{U} \subseteq 0$, where $\bar{U}$ is compact. Then $f^{-1}(\bar{U}) \cap[X \backslash 0]=F$ is compact. $p \in\left(f^{-1}(U) \backslash F\right) \cup U \subseteq 0$.

In summary, our construction yields no more, as far as compactification methods are concerned, than the method of Steiner-Steiner. Its principal value is that it unifies several apparently diverse ideas. It is clearly a generalization of Engelking's extension of the original construction. More significantly, it describes the topologies in the Steiner-Steiner example and in Whyburn's Unified Space in such a way that these two seemingly different constructions are easily seen to be equivalent. We also get the one-point compactification as a (perhaps unexpected) special case.

\section{REFERENCES}

1. P. S. Alexandroff and P. Urysohn, Mémoire sur les espaces topologique compacts, Verh. Nederl. Akad. Wetensch. Afd. Naturk. Sect. I 14 (1929), 1-96.

2. George L. Cain, Compact and related mappings, Duke Math. J. 33 (1966), 639-645.

3. George L. Cain, Richard E. Chandler and Gary D. Faulkner, Singular sets and remainders, Trans. Amer. Math. Soc. (to appear).

4. R. Engelking, On the double circumference of Alexandroff, Bull. Acad. Polon. Sci. Sér. Sci. Math. Astronom. Phys. 16 (1968), 629-633.

5. A. K. Steiner and E. F. Steiner, Compactifications as closures of graphs, Fund. Math. 63 (1968), 221-223.

6. G. T. Whyburn, A unified space for mappings, Trans. Amer. Math. Soc. 74 (1953), 344-350.

7. ___ Compactifications of mappings, Math. Ann. 166 (1966), 168-174.

Department of Mathematics, North Carolina State University, Raleigh, North Carolna 27650 